\title{
Análisis de los Ambientes virtuales para el diseño de una propuesta de interfase que sirva como herramienta en el desarrollo de proyectos de investigación
}

\author{
Mario Gerson Urbina Péreza , Josué Deniss Rojas Aragón', Omar Eduardo Sánchez \\ Estrada $^{\mathrm{c}}$ \\ ${ }^{a}$ Universidad Autonoma del Estado de México, Toluca, México, gerurb@suv.udg.mx, ${ }^{\mathrm{b}}$ Universidad \\ Autonoma del Estado de México, Toluca, México, denissrojas@gmail.com, ${ }^{\mathrm{c} U n i v e r s i d a d ~ A u t o n o m a ~}$ \\ del Estado de México, Toluca, México, omarseuaem@yahoo.com.mx.
}

\section{Resumen}

En el contexto de las universidades públicas y privadas, la investigación en Diseño Industrial no se ha destacado a nivel de otras disciplinas, en el caso particular de la Universidad Autónoma del Estado de México y sus escuelas donde se imparte el curso de Diseño Industrial: Toluca, Zumpango y Valle de Chalco, el área de investigación está por debajo de los estándares institucionales y otras disciplinas (UAEMéx Agenda estadística, 2015).

De acuerdo con las estadísticas de varios organismos de evaluación acreditados, certificados y reconocidos para el Área de Diseño Industrial en México, como ANUIES, CIEES y COMAPROD, entre los factores que más influyen para no mejorar el desempeño de la investigación de diseño se encuentran: la falta de proceso de investigación organizado; falta de herramientas digitales para la gestión de recursos; e ignorancia del proceso de investigación. Entre varios investigadores sobre el tema, destacar las contribuciones de Margolin (2005) menciona que uno de los desafíos particulares que enfrenta la comunidad de investigadores en diseño es aceptar e incluir especialistas ubicados dentro de diferentes tradiciones disciplinarias, esto no permite seguir avanzando en la búsqueda de nuevas formas de representación de diseño, por lo que el área permanece sumergida en proyectos, formas y aspectos ya existentes al intentar diseñar nuevos objetos, sin generar mayores contribuciones / contribuciones al diseño y mucho menos al proceso de investigación.

Palabras clave: investigación, enfoques metodológicos, interfaces, multidisciplinar. 


\begin{abstract}
In the context of public and private universities, research in Industrial Design has not excelled at the level of other disciplines, in the particular case of the Autonomous University of the State of Mexico and its schools where the Industrial Design course is taught: Toluca, Zumpango and Valle de Chalco, the research area is below the institutional standards and other disciplines (UAEMéx University Statistics Agenda, 2015).

According to the statistics of several accredited, certified and recognized evaluating bodies for the Industrial Design Area in Mexico, such as the ANUIES, CIEES and COMAPROD, among the factors that most influence not to improve the performance of design research are: the lack of an organized research process; lack of digital tools for resource management; and ignorance of the research process.

Among several researchers on the subject, highlight the contributions of Margolin (2005) mentions that one of the particular challenges facing the community of researchers on design is to accept and include specialists who are located within different disciplinary traditions, this does not allow to follow advancing in finding new forms of design representation, so the area remains submerged in projects, forms and aspects already existing when trying to design new objects, without generating greater contributions / contributions to the design and much less to the research process.
\end{abstract}

Keywords: research, Methodological approaches, interfaces, multidisciplinary

\title{
1. Introducción
}

En México la investigación en diseño es un gran reto, Margolín (2005) referenciado por salinas (2007), ya que si bien es cierto que existen muchas formas de diseñar, metodologias y enfoques diseñisticos, la mayoría de ellos se quedan cortas o inconclusas ya que no existen investigaciones previas que sustenten lo que se está realizando para obtener un resultado integral y que garantice que será un producto de calidad en el mercado (calidad no solo en el ámbito estético o funcional, si no enmarcando otros aspectos como; el objetivo, mensaje expresivo, durabilidad, consecuencias ambientales, seguridad al consumidor, etc), siendo estos aspectos fruto de una ardua investigación previa, de esta forma lograr así un resultado favorable para el principal objetivo que son los usuarios. 
En caso particular de algunas áreas del diseño como lo son: el rediseño de productos, la invención, construcción y el dibujo se utiliza muy poco la investigación organizada, esto se debe al pensamiento poco razonable de que "el diseño puede ocurrir sin necesidad de una investigación", sin embargo no solo basta con practicar el diseño, como ya se había mencionado anteriormente, falta encontrar la verdadera identidad del diseño dentro del país y esto no se lograra si no existe desde la raíz una verdadera investigación de los componentes culturales y antropológicos que nos lleven a detectar que es lo que no está permitiendo que se tenga una visión clara de lo que verdaderamente es el diseño industrial y de qué manera afecta o influye en la vida cotidiana.

De aquí que el uso de los ambientes virtuales son una gran herramienta para mejorar el proceso de investigación en diseño, por lo tanto en parte de esta trabajo se comentará la relevancia que tiene no solo para la disciplina del diseño industrial, sino para la investigación en el contexto nacional.

\section{Antecedentes}

Se considera a un ambiente virtual de aprendizaje como el espacio físico donde convergen las nuevas tecnologías para desarrollarse y potencializarse y rebasar aquellos entornos escolares tradicionales, y que a su vez favorecen al conocimiento (Avila y Bosco 2001) y a la apropiación de contenidos, experiencias y procesos pedagógico-comunicacionales; creando nuevas escenarios vistos como formas de trabajo, y de interacción entre los usuarios, en proyectos vanguardistas que crean ambientes educativos innovadores, nuevas experiencias de aprendizaje, pero sobretodo que son poderosas herramientas para la diversificación de aplicaciones actividades académicas y de investigación, sobre esto último se verá la importancia que tienen los ambientes virtuales y su relación con el proceso de investigación.

Para Samaja (2002), las universidades tienen una postura bien definida y precisa respecto al tipo de investigación que deben promover y el porque la realizan, y para él gran parte del porque tiene que ver con la palabra ciencia, independientemente de su enfoque y de sus acepciones, este autor define cuatro tipos:
A. Ciencia como los saberes tradicionales.
B. Ciencia como saber reflexivo.
C. Ciencia como conocimiento obtenido.
D. Ciencia como investigación destinada. 
Después de esta breve semblanza, es obvio que la que merece atención prioritaria para cada universidad y dependiendo del contexto institucional, nacional e internacional es la investigación destinada a la innovación tecnológica; Todo lo anterior indica que las universidades que quieren hacer investigación deben de adaptarse a los tiempos y contextos actuales, pero las condiciones son muy diferentes en estos ámbitos, por lo tanto el nivel de desarrollo no puede ser el mismo para todos; diversos autores e investigadores mencionan que es necesario trabajar en estos cuatro grandes pilares:

1) Formar a los individuos en una percepción de la realidad inspirada en una actitud protagónica, que se nutra de todas las riquezas creadas por la evolución y la historia de la humanidad, promoviendo una formación estética que incluya la ética, la reflexión, la comprobación productiva y la vocación innovadora.

2) Formar profesionales orgullosos de las tradiciones más actualizadas y consensuadas por la comunidad de pares, y con la capacidad para aplicarlas con creatividad en el estricto marco de la ética de su corporación profesional.

3) Formar docentes universitarios calificados con lo más altos estándares académicos, capaces de expandir las fronteras de los conocimientos en su disciplina, de realizar síntesis de profundo valor reflexivo que mantengan vivos los ideales regulativos de la razón en su campo disciplinario particular, y abierta la reflexión interdisciplinaria y transdisciplinaria.

4) Formar investigadores experimentales rigurosos, capaces de someter al control de los hechos mediante diseños imaginativos.

Para la Universidad Autonoma del Estado de México (UAEMéx) la investigación es una función sustantiva que sostiene y da razón de ser a esta institución, junto con la docencia y la extensión definen sus identidad esencial, atendiendo a los preceptos de humanismo y filosofía que sirven como base para la construcción de una ética universitaria comprometida con la responsabilidad social.

A través de ella, se pueden lograr objetivos fundamentales, tales como:

- Fortalecer la producción de conocimiento científico.

- Favorecer la formación de una comunidad académica (docentes, investigadores y alumnos) con mentalidad proactiva en la busqueda de soluciones, aplicando conocimientos y trabajo multidisciplinario.

- Integrar redes de colaboración promoviendo el desarrollo y la innovación.

- Ofrecer un servicio real y de utilidad a la sociedad. 
La investigación y el desarrollo (sus siglas I+D) comprenden el trabajo creativo llevado de forma sistemática para la generación de líneas de generación y aplicación del conocimiento. Para la UAEMéx el desarrollo científico tecnológico representa un instrumento indispensable para el avance social. Las universidades representan el espacio natural para el desarrollo y fortalecimiento de la producción de conocimiento científico, ya que es en ellas donde se deben generar soluciones creativas e innovadoras para las innumerables áreas del quehacer social. En este sentido para la UAEMéx la investigación es un área estratégica para el avance del Estado de México y del propio país.

Técnicamente un proyecto de investigación se define como el conjunto de actividades de ID+i a realizarse por un equipo idóneo de personas (de preferencia tipo interdisciplinario), en un lugar y periodo determinado, con determinados recursos y condiciones para lograr objetivos y metas preestablecidas; atendiendo una planeación minuciosa de métodos, estrategias y herramientas para desarrollar la alternativa de solución seleccionada ante un problema de carácter científico, técnico o tecnológico.

Los proyectos académicos de investigación se deben caracterizar por la calidad, tanto de la información, como de las decisiones. La garantía de esa calidad es el profesor-investigador, quien debe ser especialista en el área de la ciencia o la tecnología a la cual se adscribe el problema, experto en la aplicación de los métodos y técnicas del área, acucioso en la tanto del problema, como de la alternativa de solución y exigente con la calidad de la información que respalda el problema y las decisiones del proyecto.

Gestión de Proyectos Académicos.

Para diferentes autores, la gestión de proyectos es el proceso organizado y sistemático en donde se organiza y administran los recursos de tal forma que se alcancen los objetivos y generen los productos de un proyecto en tiempo y forma, caso contario los proyectos de investigación académicos se diferencian de otros tipos de proyectos por abordar problemas no rutinarios, con técnicas y procedimientos no estandarizados, buscando resultados nuevos y novedosos. Estas características los convierten en proyectos con un alto grado de incertidumbre y riesgo; hecho que demanda procesos continuos y minuciosos de planificación, organización, seguimiento y control.

\section{Propósito}

Anteriormente se discutió la importancia que tiene a nivel institucional y nacional la investigación, pero aunque pareciera que se debió de empezar con el status a nivel mundial, cabe señalar que es un contexto recíproco, ya que el diseño industrial forma parte 
indispensable del desarrollo económico y social de la mayoria de los paises, las propias caracteristicas del diseño le permiten idear y desarrollar productos y servicios que logren cubrir las necesidades de los clientes y consumidores, o bien aportando mejoras a los productos ya existentes o a los futuros, pero a pesar de tantas bondades como disciplina a nivel internacional, el Diseño en México se ha limitado en la etapa de "construir objetos", dejando fuera un factor indispensable como lo es la investigación.

Por ello cuando se escucha el término de investigación en diseño, inmediatamente se piensa en acciones tales como consultorías, trabajo de oficina (papeleo), conferencias, trabajo en salas de lectura, asistencia a bibliotecas, etc. una de las razones por lo que posiblemente esto ocurre, es por la asociación de la palabra diseño al contexto de la creatividad, innovación y visión futurista, mientras que para muchos la investigación significa rigor y evidenciar que realmente existe; de primera instancia parecería que los dos términos se contraponen porque van en caminos diferentes , pero esta "contraposición" crea un espacio muy interesante de interpretación y adaptación para la actividad del diseñar, lo que genera nuevas oportunidades para que la disciplina siga creciendo en diferentes ambientes y contextos.

\section{Metodo}

Una investigación según Sampieri (2010) se define como un conjunto de procesos sistemáticos, críticos e inclusive empiricos que se aplican al estudio de ciertos fenomenos y problemas. Según la clasificación de este autor quién divide la investigación en tres tipos que son: investigación aplicada (de las mas utilizadas en el diseño), investigación cientifica, e investigación y desarrollo (en esta última es donde el diseño deberia de participar en mayor proporción, ya que su propósito es desarrollar productos y/o servicios que justifiquen las propuestas generadas). Es por ello que uno de desafíos particulares que enfrenta el metodo de esta investigación en dos momentos en particular: al principio en analizar como la comunidad de investigadores sobre diseño dentro del enorme contexto de distintas tradiciones disciplinarias, avanza en encontrar nuevas formas de investigar para la representación del diseño (hacer investigación), y posteriormente en como la carencia de herramientas para lograr una optima investigación genera que los resultados sigan sumergidos en proyectos, formas y aspectos ya existentes al momento de tratar de diseñar nuevos objetos, sin dar mayores aportaciones al diseño y mucho menos a la parte de investigación. 


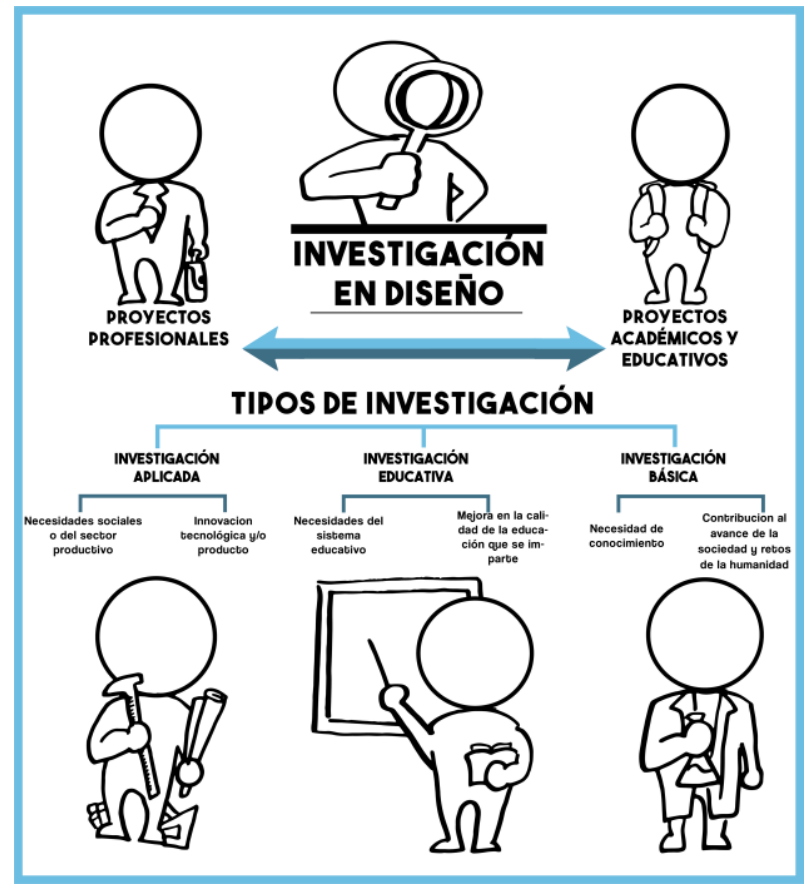

Esquema 1.1. resultados sobre investigación en Diseño en la UAEMéx Fuente: Elaboración propia (2019)

\section{Objetivo}

Analizar el proceso de investigación en diseño y explotar las posibilidades de la generación de futuras herramientas que involucren información sobre el binomio de diseñoinvestigación, y que sean capacesde enfrentar con mayor certeza temas especificos y problemas de indole profesional.

\section{Resultados}

De todo lo anterior hay que puntualizar que la investigación sin una previa, bien planteada y justificada metodología no podría estar encaminada correctamente. Para Margolin (2005), el proceso metodologico es considerado como una parte crucial en el desarrollo de cualquier tipo de investigación, ya que indica los pasos a seguir, las diferentes etapas del 
proceso de trabajo, el dominio y manejo de las teorías más importantes o los conceptos más relevantes de la investigación que serán el sustento principal de todo proyecto.

Es por ello que después de analizar el contexto de diferentes instituciones sobre los principales obstaculos y oportunidades que tiene el diseño Industrial y el proceso de investigación en Mèxico, de ser bien afrontados estos obstaculos y ser aprovechadas las oportunidades se perciben nuevos escenarios a futuro dentro del contexto nacional, estatal e institucional siendo el caso de la UAEMéx (Tabla 1.1).

Tabla 1.1. resultados sobre investigación en Diseño en la UAEMéx

\begin{tabular}{|c|c|c|}
\hline Organismo & Obstáculos & Oportunidades \\
\hline CIEES & $\begin{array}{l}\text { - Falta de un proceso de } \\
\text { investigación establecido para la } \\
\text { licenciatura. } \\
\text { - Falta del uso de ambientes } \\
\text { virtuales e instrumentos digitales. }\end{array}$ & $\begin{array}{l}\text { - Establecer bases para la generación } \\
\text { de un método, metodología o } \\
\text { enfoque específico de la } \\
\text { licenciatura que contribuya a crear } \\
\text { una identidad en el área de } \\
\text { investigación. }\end{array}$ \\
\hline COMAPROD & $\begin{array}{l}\text { - Falta de infraestructura para } \\
\text { generar investigación. } \\
\text { - Falta de sitios especializados para } \\
\text { publicar. } \\
\text { - Alto costo de las publicaciones. }\end{array}$ & $\begin{array}{l}\text { - Generar más productos sobre } \\
\text { investigación en diseño. } \\
\text { - Elevar el nivel de las publicaciones. } \\
\text { - Obtener mayores recursos para el } \\
\text { área y proyectos. }\end{array}$ \\
\hline ANUIES & $\begin{array}{l}\text { - Falta del uso de ambientes } \\
\text { virtuales e instrumentos digitales. }\end{array}$ & $\begin{array}{l}\text { - Utilizar temas innovadores basados } \\
\text { en las herramientas digitales y } \\
\text { difundir en espacios especializados. }\end{array}$ \\
\hline SIEA (organismo Interno UAEMéx). & $\begin{array}{l}\text { - No se cuenta con información } \\
\text { precisa sobre el tema. }\end{array}$ & $\begin{array}{l}\text { - Generar bases de datos sobre la } \\
\text { carrera y sus núcleos de } \\
\text { información. }\end{array}$ \\
\hline
\end{tabular}

Fuente: Elaboración propia (2019) 


\section{Conclusiones}

- En México la investigación en diseño es un gran reto, ya que si bien es cierto que existen muchas formas de diseñar, metodologias y enfoques diseñisticos, la mayoría de ellos se quedan cortas o inconclusos ya que no existen investigaciones previas que sustenten lo que se está realizando para obtener un resultado integral

- Los resultados previos demuestran la necesidad de apoyar el proceso de investigación en Diseño industrial, ya que esto aumentará el número de investigadores de la disciplina en la UAEM, es por ello que los ambientes virtuales y digitales juegan un papel importante en este proceso.

- El uso de la realidad virtual, las animaciones, modelados digitales son importantes herramientas que ayudarán a crecer el proceso de investigación.

- $\quad$ Es indispensable que existan lugares apropiados y accesibles para poder compartir y difundir los resultados de las investigaciones en diseño, porque a la fecha son muy pocos además de costosos.

- los diseñadores desde su etapa como estudiantes (para efectos de esta investigación se tomará en cuenta la etapa de formación académica universitaria), se enfrentan a la paradoja de la investigación sin saber como realizarla y mucho menos aplicarla.

\section{References}

Guzmán, Jesús Carlos. (2011). La calidad de la enseñanza en educación superior ¿Qué es una buena enseñanza en este nivel educativo?. Perfiles educativos, 33(spe), 129141. Recuperado en 08 de octubre de 2019, de

http://www.scielo.org.mx/scielo.php?script=sci_arttext\&pid=S018526982011000500012\&lng=e $\&$ tlng=es.

Margolin (2005) Las rutas del diseño: Estudios sobre teoría y práctica primera edición, México: Designio.

Patricia Avila M. Martha Diana Bosco H. (2001). Ambientes virtuales de aprendizaje una nueva experiencia. 09 octubre 2019, de 20th International Council for open and distanceeducationSitioweb:http://investigacion.ilce.edu.mx/panel_control/doc/c37ambientes.pdf

Samaja, J. (2002) Epistemología y metodología: Buenos Aires: Eudeba.

Sampieri (2010) Metodología de la investigación Quinta Edición, Mc Graw Hill.

Universidad Autónoma del Estado de México, Agenda Estadistica 2015, http://planeacion.uaemex.mx/docs/AE/2015/AE_2015.pdf 